\title{
SULLA INDIVISIBILITÀ DEI DIRITTI FONDAMENTALI
}

\author{
Arnaldo Miglino*
}

RESUMO: O autor enfrenta os direitos fundamentais partindo da noção de indivisibilidade dos direitos, negando a perspectiva de gerações ou dimensões, em busca de uma maior efetividade, com a rejeição da lógica dos custos decorrente da análise econômica do direito.

Nella tradizione giuridica degli stati democratici, i diritti che il cittadino può vantare nei confronti dei poteri pubblici sono distinti in civili, economici, politici e sociali. La suddivisione è ben consolidata e grosso modo ${ }^{1}$ corrisponde alle varie fasi storiche, dalla nascita del liberalismo sino ai nostri giorni, che hanno visto trasformare il rapporto fra governanti e governati. Con la creazione dello stato liberale l'individuo rivendica un ambito di libertà e di indipendenza nei confronti del potere, cui è imposto il rispetto della libertà personale, religiosa, di

* Avvocato cassazionista e docente universitario nella Università degli Studi di Roma "La Sapienza".

${ }^{1}$ Alcuni diritti civili (si pensi alla libertà di associazione e di riunione) si sono consolidati nel momento storico in cui si sono affermati i diritti sociali; $i$ diritti politici, caratterizzanti l'ordinamento democratico, esistevano solo per ristrette classi di cittadini già nel periodo liberale; certi diritti sociali (si pensi agli emolumenti previdenziali nella Germania di Bismarck) sono nati quando ancora non si erano pienamente affermati i diritti politici. manifestazione del pensiero. Nascono così i diritti civili, attraverso i quali si definisce una sfera individuale nella quale il cittadino pretende che lo stato non si intrometta. In seno ai diritti civili si distinguono i diritti economici, che garantiscono la libertà di azione economica attraverso gli istituti della proprietà e del contratto. Diritti civili ed economici rappresentano una conquista della borghesia contro il vecchio ordine ereditato dall'età medievale e, in quanto mirano ad impedire l'ingerenza statale nei confronti dell'individuo, sono detti anche diritti di libertà dallo stato. Per indicare la loro collocazione storica sono chiamati diritti di prima generazione, essendo le prime posizioni soggettive pubbliche ad essere rivendicate nel cammino che ha condotto dallo stato liberale a quello sociale.

Nello stato liberale solo pochi possidenti avevano il controllo della politica, in quanto il suffragio non era universale, non era libero l'associazionismo sindacale e politico, il 
diritto di riunione non godeva di sufficienti garanzie. Per effetto di mutamenti che prendono l'avvio dalle rivendicazioni della classe operaia, il diritto di voto è esteso a tutti i componenti della collettività che ottengono, altresì, il diritto di riunirsi e di associarsi, di formare partiti e sindacati. Tali diritti, detti anche di seconda generazione, sono politici perché consentono a tutti i cittadini di partecipare alla vita politica e di svolgere la loro libertà nello stato che, pertanto, non è più soltanto liberale ma diventa democratico.

Per effetto degli impulsi derivanti dalla più ampia partecipazione popolare agli assetti politici ed istituzionali, nasce una terza generazione di diritti che, al contrario dei diritti di libertà, anziché impedire intrusioni del potere nella sfera esistenziale individuale, lo obbligano a fornire al cittadino utilità ed opportunità. Si tratta dei diritti sociali: il diritto alla salute con cure gratuite agli indigenti, all'istruzione, all'assistenza ed alla previdenza sociale. La realizzazione di questi diritti fa sì che determinate libertà vengano assicurate mediante lo stato, che non si limita ad essere liberale e democratico, ma diviene sociale in quanto cura il benessere della società.

Alle diverse dinamiche storiche che hanno determinato i diritti pubblici fanno capo le classificazioni di tipo più strettamente tecnico-giuridico. I diritti, o libertà, di tipo civile ed economico sono definiti come libertà negative o diritti negativi. Con ciò si vuole intendere che la soddisfazione della posizione soggettiva è assicurata attraverso la non ingerenza del potere pubblico: la realizzazione dell'interesse protetto dal diritto soggettivo avviene attraverso un dovere di astensione.
I diritti sociali sono definiti come libertà positive o diritti positivi. Con essi il cittadino reclama l'intervento dei pubblici poteri per la soddisfazione dell'interesse protetto dalla posizione giuridica soggettiva, che assume i connotati della pretesa.

Così, i diritti negativi vengono modellati secondo lo schema tipico del diritto di proprietà, come situazioni soggettive capaci di far godere al singolo un bene della vita attraverso la mera astensione degli altri consociati. I diritti positivi si configurano secondo lo schema dei diritti di credito, quali situazioni soggettive la cui realizzazione è legata ad una specifica prestazione da parte di un soggetto determinato. Da tale concezione sembra emergere una differenza qualitativa fra le libertà negative e quelle positive: queste ultime, al contrario delle prime, possono essere realizzate solo con interventi che comportano spese gravanti sulla collettività. Il pensiero neo-liberale contesta l'interventismo pubblico nella società civile e propugna un ritorno al cosiddetto 'stato minimo', che garantisca in maniera compiuta solo le libertà negative e receda dalla tutela dei diritti sociali. Tale posizione viene giustificata, da un lato, dalla constatazione dei maggiori costi che comportano per la collettività le libertà positive; dall' altro dalla credenza che il modo migliore per risolvere i problemi della società sia quello di affidarli alla logica del sistema di mercato, storicamente vincente. La concezione di fondo che sottende un tale modo di vedere le cose è quella secondo cui la libertà del cittadino dai pubblici poteri è soprattutto libertà dal potere impositivo fiscale. Poiché le spesa necessaria ad assicurare la soddisfazione dei diritti positivi è finanziata attraverso il 
prelievo coattivo delle risorse dei singoli, la liberazione di cittadini dalle imposte deve passare attraverso una riduzione delle libertà positive. Ciò non porterebbe ad un peggioramento complessivo della vita sociale perché essa, affidata all'efficienza del sistema di mercato, libererebbe le energie migliori evitando sprechi e forme di parassitismo. Per esprimere in maniera estremamente sintetica l'avversità allo stato sociale e l'auspicio di uno stato minimo, si possono ricordare le parole di Charles Murray (1997, p. 5), secondo cui "un uomo che si guadagna onestamente da vivere e che si fa i fatti propri merita di essere lasciato in pace", e di David Boaz (1997, p. 12) secondo il quale "il problema vero negli Stati Uniti è lo stesso di tutto il mondo: troppo stato".

Sia l'enfatizzazione del costo delle libertà positive che l'ottimistica fiducia nella capacità del mercato non sono condivisibili. Affrontiamo con ordine le due questioni.

In una recente opera, Stephen Holmes e Cass R. Sunstein (1999-2000) hanno mostrato come la tutela di tutti i diritti, sia positivi che negativi, ha un costo che ricade sulla collettività. Certamente, nelle partite contabili di bilancio, sarà più facile individuare quanto i pubblici poteri spendono per assicurare il diritto alla salute, o all'istruzione, che quanto spendono per assicurare le libertà negative, ma non per questo si può assumere che queste ultime non comportano una spesa pubblica. Il cittadino che vuole essere lasciato in pace dallo stato, soprattutto dal fisco, non si avvede che, in tanto riposa tranquillamente nella sua villetta, beandosi di un importante affare che sta andando in porto, in quanto è piuttosto improbabile che: il primo malintenzionato lo cacci dalla sua abitazione; questa sia divorata da un incendio; un aereo, persa la rotta ed andando a collidere con altro veivolo, si schianti sulla casa; giunga la notizia che l'affare è sfumato perché il contratto non è stato tenuto in nessun conto dall' altro contraente. Tutto ciò è improbabile perché i pubblici poteri hanno istituito e mantengono, e quindi pagano: le forze dell'ordine, i vigili del fuoco, il servizio radar, gli uffici giudiziari. Se poi lo stesso cittadino, dopo aver goduto, nella sua bella proprietà immobiliare, della soddisfazione ricevuta dall'affare andato in porto grazie alla sua libertà negoziale, decidesse di fare una passeggiata per godere della sua libertà negativa di circolare liberamente, potrebbe usufruire del marciapiedi costruito, tenuto in efficienza ed illuminato a spese dall'amministrazione comunale....

E' ben evidente che la soddisfazione dei diritti negativi, come di quelli positivi, dipende dall'erogazione di servizi, da prestazioni da parte dei poteri pubblici. A seconda del contenuto del diritto variano le tipologie delle prestazioni destinate a garantirlo, ma tutte sono effettuate spendendo risorse. Anche i diritti politici hanno bisogno di mezzi per essere goduti: si pensi alle strutture necessarie per una consultazione popolare. Come hanno un costo gli apparati per assicurare il diritto all'istruzione o il diritto alla salute, così hanno un costo gli apparati che consentono l'esercizio della libertà negoziale e la fruizione dei beni rientranti nella sfera giuridica individuale, o l'esercizio di libere elezioni. Maggiore sarà la spesa efficientemente destinata ad una funzione pubblica, migliore sarà la qualità ed il livello di tutela del diritto che la funzione è tesa a soddisfare. Ciò è vero sia per i diritti 
pubblici positivi che per quelli negativi. Infatti, come il diritto alla salute tanto meglio si realizza quante più prestazioni sanitarie vengono erogate al cittadino, anche la libertà negoziale, o il diritto di proprietà, sono resi tanto più sicuri quanto più sono potenziati gli apparati giudiziari e di ordine pubblico.

Tenuto conto di ciò, dire che lo stato deve ritornare 'minimo' significa, in sostanza, assumere non già che gli apparati pubblici devono astenersi dallo sperperare risorse collettive, ma che le risorse collettive devono essere spese solo per soddisfare alcune categorie di interessi anziché altre. Preso atto di questa realtà, si demistifica il discorso neo-liberale e lo si valuta per quello che esso è: il perseguimento di un modello di organizzazione sociale, storicamente superato, in cui le risorse della collettività sono destinate a soddisfare in via preferenziale i bisogni della classe agiata. Poiché tutti i diritti pubblici hanno un costo collettivo - per dirla con gli autori citati, "tutti i diritti sono diritti positivi" e "la tutela dei diritti dipende in realtà dalle attuali aliquote d'imposta" (Holmes e Sunstein, 1999-2000, p. 37 e 31) -, le discussioni sull'eventualità di una maggiore o minore estensione delle libertà a seconda del loro carattere civile o sociale perdono significato se non si tiene conto della reale posta in gioco: la scelta degli interessi che la collettività deve soddisfare con le risorse che è disposta a spendere.

L'altro argomento caro ai fautori dello stato minimo, l'efficienza del sistema del libero mercato, fondato sul principio secondo cui il miglior modo per raggiungere l'interesse comune è che ogni individuo persegua il suo interesse individuale, è un non senso dal punto di vista della teoria dei diritti. Come ha avuto modo di sostenere proprio uno dei maggiori esponenti della finanza mondiale (Soros 2000-2001, p. 10, $11,89,155,158)$, il mercato riguarda solo il mondo dei valori economici ove ogni soggetto ha l'unico scopo di massimizzare il suo profitto, ma non è capace di dare risposte ad altri bisogni collettivi che non attengono ai rapporti di scambio: "pace e sicurezza, legge e ordine, diritti umani, protezione dell' ambiente e alcuni elementi di giustizia sociale" (Soros cit. p. 11). Ciò è senz'altro da condividere. Il sistema delle libere transazioni economiche nessuna indicazione utile può dare in materia di diritti fondamentali, alla quale è del tutto alieno. Le libertà, negative o positive che siano, riguardano il mondo dei valori sociali e si realizzano non mediante scambi ritenuti convenienti dai soggetti che possono parteciparvi, ma attraverso la previsione ed il rispetto di doveri, ossia di comportamenti che realizzano un sacrificio da parte di chi li compie. Non ha senso parlare della bontà e dell'efficienza del sistema di mercato nel regolare i rapporti sociali, perché questi trovano il loro punto di equilibrio non già ove si incontrano le volontà di chi offre e di chi acquista, ma nella volontà normativa di imporre determinati sacrifici di interessi (doveri) per assicurare il soddisfacimento di altri interessi valutati come prevalenti rispetto ai primi (diritti). E' evidente che i bisogni della collettività di tipo non economico non possono essere risolti con la logica economica del mercato. Certamente, come si è già visto, le posizioni soggettive pubbliche hanno una forte rilevanza economica perché impongono l'erogazione di risorse, ma non hanno una 
finalità economica. In altri termini, le libertà hanno non già lo scopo di realizzare un risultato economico secondo la logica del profitto, ma un dato assetto sociale, che ovviamente comporta dei costi.

Dal discorso sin qui svolto emerge che non è possibile attribuire alcuna valenza ontologica alla classificazione dei diritti pubblici: tutti esprimono valori e interessi da soddisfare mediante prestazioni il cui costo deve essere sostenuto dalla collettività. Né si può pensare che questi interessi possano essere perseguiti dal sistema di mercato che, per sua stessa struttura, assume un valore unico e peculiare: il profitto. Bisogna riconoscere che tutti i diritti pubblici sono fondamentali ed indivisibili nel senso che sono necessari, nel loro complesso, per realizzare valori che assicurino al cittadino dignità, rispetto e sviluppo della personalità. Riguardo all' ordinamento costituzionale italiano si è già sottolineato (Mazziotti 1993, p. 56) come i diritti fondamentali cui si riferisce l'art. 2 della Costituzione siano tutti quelli previsti dalla prima parte della costituzione stessa che disciplina, per l'appunto, i diritti civili, sociali, economici e politici. Ma il concetto di indivisibilità dei diritti emerge in maniera forte dalla struttura della Carta dei diritti fondamentali dell'Unione europea proclamata dal Consiglio europeo riunito a Nizza dal 7 al 9 dicembre $2000^{2}$. Il documento, nell'enunciare i diritti riconosciuti dall'Unione, li riunisce nell'ambito di sei valori: dignità ${ }^{3}$, libertà ${ }^{4}$,

\footnotetext{
${ }^{2}$ D'ora in poi si farà riferimento a tale documento indicandolo con il termine 'Carta'.

${ }^{3}$ Capo I, artt. 1-5.

${ }^{4}$ Capo II, artt. 6-19.
}

uguaglianza $^{5}$, solidarietà ${ }^{6}$, cittadinanza ${ }^{7}$, giustizia $^{8}$. Nel capo II dedicato alla libertà, troviamo non solo la libertà d'impresa ${ }^{9}$ ed il diritto di proprietà ${ }^{10}$, ma anche il diritto all'istruzione di cui all' art. $14 \mathrm{che}$, al comma $2^{\circ}$, sancisce "la facoltà di accedere gratuitamente all'istruzione obbligatoria". Come si vede, il principio di libertà comprende sia le classiche 'libertà negative' conquistate dal liberalismo borghese, che un'importante 'libertà positiva' ottenuta dalle classi meno abbienti. Nella costituzione italiana la proprietà e la libertà d'impresa sono disciplinate nel titolo attinente ai diritti economici, mentre l'istruzione gratuita è garantita nel titolo riguardante i diritti sociali. Tale schema, indicativo dell'origine storica delle diverse posizioni soggettive, è del tutto assente nella Carta, ove le situazioni soggettive sono accomunate dal valore che attraverso esse deve essere perseguito. Si consideri ancora che, nel capo III della Carta dedicato all'uguaglianza, oltre a disposizioni che richiamano il principio dell'uguaglianza formale ${ }^{11}$, troviamo disposizioni che contemplano "misure che prevedono vantaggi specifici a favore del sesso sottorappresentato" 12 ed altre forme di intervento nei confronti di bambini, anziani, disabili, che nella costituzione italiana corrispondono all'esercizio di diritti sociali.

\footnotetext{
${ }^{5}$ Capo III, artt. 20-26.

${ }^{6}$ Capo IV, artt. 27-38.

${ }^{7}$ Capo V, artt. 39-46.

${ }^{8}$ Capo VI, artt. 47-50.

${ }^{9}$ Cfr. art. 16.

${ }^{10} \mathrm{Cfr}$. art. 17.

${ }^{11} \mathrm{Cfr}$. artt. 20, 21, 23 comma $1^{\circ}$.

${ }^{12}$ Cfr. art. 23 comma $2^{\circ}$.
} 
Nel capo IV che la Carta dedica alla solidarietà si trovano sia diritti che, come i diritti civili della tradizione liberale, sono garantiti dagli apparati giudiziari e di ordine (diritto di negoziazione e di azioni collettive dei lavoratori e dei datori di lavoro ${ }^{13}$, tutela in caso di ingiustificato licenziamento ${ }^{14}$ ), che i diritti alla protezione della salute ${ }^{15}$, alla sicurezza ed all'assistenza sociale ${ }^{16}$, che necessitano di apparati erogatori di specifici servizi.

Si vede bene che, secondo l'impostazione con la quale la Carta è stata redatta, la distinzione tra diritti positivi e negativi, civili e sociali, rimane priva di senso, perché tutti i diritti sono strumenti necessari, e quindi interdipendenti e connessi, per perseguire dei valori. Dalla lettura della Carta emerge che l'individuo non è libero non solo se non può negoziare ed ottenere la proprietà, ma anche se rimane privo di una istruzione di base. Non vi è uguaglianza per bambini, anziani e disabili e sesso debole che, pur non essendo discriminati, rimangono privi di prestazioni idonee a compensare le iniziali situazioni di svantaggio. Non vi è solidarietà se, oltre alle magniloquenti affermazioni di principio, non si predispongono servizi per garantire la sicurezza sociale e la salute di chi non potrebbe provvedere con i suoi soli mezzi alle difficoltà della vita. La scelta operata dalla Carta può essere condivisa o non condivisa sul piano etico, ma bisogna riconoscere che dal punto della teoria giuridica è senz'altro

\footnotetext{
${ }^{13}$ Cfr. art. 28.

${ }^{14}$ Cfr. art. 30 .

${ }^{15}$ Cfr. art. 35 .

${ }^{16}$ Cfr. art. 34.
}

un passo avanti. Infatti, la visione dei diritti fondamentali come posizioni giuridiche strumentali alla realizzazione di valori, abbandonando la tradizionale distinzione che si riporta all'origine storica delle libertà, ne chiarisce i contenuti, i presupposti e le condizioni per l'esercizio e, inoltre, supera le contraddizioni insite nella stessa teoria liberale, acutamente evidenziata dal Rodotà (2001, p. 83 e 84), il quale afferma che "se, come questa teoria vuole, v'è un nesso inscindibile tra libertà e proprietà, la mancanza in un soggetto dei mezzi necessari per una vita dignitosa costituisce una menomazione della sua libertà, aprendo così la strada alla necessaria considerazione anche di politiche pubbliche attive, volte ad eliminare questa condizione inaccettabile".

\section{BIBLIOGRAFIA}

1) Holmes Stephen e Sunstein Cass R. Sunstein (1999), The Cost of Rights. Why Liberty

Depends on Taxes, New York, W.W. Norton, trad. Elisabetta Caglieri nell'edizione italiana curata da Carlo Fusaro (2000), Bologna, Società editrice il Mulino.

2) Murray Charles, What It means to be a Libertian, A personal Interpretation, (1997) New York, Broadway Books, citato in Holmes Stephen e Sunstein Cass R. Sunstein (1999), The Cost of Rights. Why Liberty Depends on Taxes, New York, W.W. Norton, trad. Elisabetta Caglieri nell'edizione italiana curata da Carlo Fusaro (2000), Bologna, Società editrice il Mulino, p. 13 e 14.

3) Boaz David (1997), Libertarianism: a Primer, New York, Free Press, 1997, citato in Holmes Stephen e Sunstein Cass R. Sunstein (1999), The Cost of Rights. Why 
Liberty Depends on Taxes, New York, W.W. Norton, trad. Elisabetta Caglieri nell'edizione italiana curata da Carlo Fusaro (2000), Bologna, Società editrice il Mulino, p. 13 e 14 .

4) Holmes Stephen e Sunstein Cass R. Sunstein (1999), The Cost of Rights. Why Liberty Depends on Taxes, New York, W.W. Norton, trad. Elisabetta Caglieri nell'edizione italiana curata da Carlo Fusaro (2000), Bologna, Società editrice il Mulino.
5) Soros George (2000), Open Society, Reforming Global Capitalism, published in the United States by PubblicAffairs TM, a member of the Perseus Book Group, traduzione italiana di Massenzio Taborelli (2001), Milano, Ponte alle Grazie s.r.1..

6) Mazziotti di Celso Manlio (1993), Lezioni di diritto costituzionale, Parte II, $2^{\circ}$ ed., Milano, Giuffrè editore.

7) Rodotà Stefano (2001), "La Carta come atto politico e documento giuridico", in Riscrivere i diritti in Europa, Bologna, Società editrice Il Mulino. 
